\title{
Factores que influyen en las diferencias entre la evaluación global de la actividad de la enfermedad por el paciente y por el médico en pacientes con artritis psoriásica
}

\author{
J. Gallino Yanzi ${ }^{1}$, O.L. Cerda ${ }^{1}$, M. Landi ${ }^{1}$, C.A. Zaffarana ${ }^{1}$, E.E. Schneeberger ${ }^{1}$, G. Citera ${ }^{1}$ \\ ${ }^{1}$ Instituto de Rehabilitación Psicofísica, CABA, Argentina
}

\section{Resumen}

Objetivo: Evaluar los factores que influyen en la evaluación global de la actividad por el paciente (EGEp) y el médico (EGEm) en pacientes con Artritis Psoriásica (APs).

Materiales y Métodos: Se incluyeron pacientes con diagnóstico de APs. Se evalúo rigidez matinal, dolor y evaluación global de la enfermedad por el paciente y el médico por escala visual análoga (EVA). Los pacientes completaron: BASDAl, HAQ-A, BASFI, ASQoL y PSAQoL. Se calcularon DAS28, DAPSA y CPDAl.

Resultados: Se incluyeron 110 pacientes. La EGEp tuvo muy buena correlación con EVA dolor (r:0,76), BASFI (r:0,7) y BASDAI (r:0,7). Por el contrario, la EGEm tuvo buena correlación con EVA dolor (r:0,65), BASDAI $(r: 0,62)$ y EGEp $(r: 0,64)$. En los análisis de regresión lineal múltiple, utilizando EGEm y EGEp como variables dependientes, el dolor se asoció significativamente a ambas (coef $\beta: 0,529, p<0,001$ y coef $\beta: 0,481, p=0,002)$. En la regresión logística, considerando MDA (Minimal Disease Activity) como variable dependiente, la EGEp tuvo una influencia mayor que la del médico [OR: 0,61 (IC 95\%: $0,42-0,89$ ), $p=0,01$ vs 0,49 (IC 95\%: 0,29-0,87), $p=0,02)]$.

Conclusión: Ambas evaluaciones presentaron buena correlación. El dolor fue la única variable que influyó independiente sobre ambas. La evaluación por el paciente tuvo mayor influencia sobre la presencia de MDA.

Palabras clave: artritis psoriásica, actividad de la enfermedad por el médico, actividad de la enfermedad por el paciente.

\section{Abstract}

Objective: To evaluate the agreement and the variables that influence global disease assessment by the patient (PGA) and physician (PhGA) in patients with PsA.

Methods: Patients with PsA were included. We calculated DAS28, DAPSA, CPDAl and MDA. Morning stiffness, pain and global disease assessment by patients and physicians were assessed using visual analogue scale (VAS 0-10 cm). Patients completed BASDAl, HAQ-A, BASFI, ASQoL and PSAQoL.

Results: 110 patients were included. The PGA had very good correlation with pain (Rho=0.76), BASFI (Rho=0.7) and BASDAl $(\mathrm{Rho}=0.7)$. By contrast, the PhGA had a good correlation with pain $(R h 0=0.65), \operatorname{BASDAl}(\mathrm{Rho}=0.62)$ and PGA (Rho=0.64). In two multiple linear regression analysis, using PhGA and PGA as dependent variables, pain was the main variable that was significantly associated with both of them ( $\beta$ coeff: $0.529, p<0.001$ and $\beta$ coeff: $0.481, p=0.002$, respectively). Taking Minimal Disease Activity (MDA), we performed a logistic regression analysis, considering MDA as a dependent variable. PGA had a greater association as compared to the PhGA [OR: 0.61 (95\% Cl: $0.42-0.89$ ), $p=0.01$ vs 0.49 (95\% Cl: 0.29-0.87), $p=0.02$.

Conclusion: Both assessments showed good correlation. Pain was the variable most strongly influencing both. PGA had a stronger association with MDA compared to PhGA.

Key words: psoriatic arthritis, physician disease activity, patient disease activity. 


\section{Introducción}

La Artritis Psoriásica (APs) es una artropatía inflamatoria que se presenta en el $5-40 \%$ de los pacientes con psoriasis cutánea ${ }^{1}$. En nuestro país, la incidencia de esta patología es de 6,26 casos nuevos cada 100.000 habitantes/año ${ }^{2}$. Afecta a personas de ambos sexos, más frecuentemente entre los 20 y 40 años. El cuadro es variado e incluye compromiso del esqueleto axial, oligoartritis asimétrica o poliartritis, dactilitis, entesitis, compromiso ungueal y manifestaciones extraarticulares ${ }^{3}$. La evaluación de la actividad de la enfermedad así como las decisiones terapéuticas se basan en los resultados reportados por los pacientes en combinación con la percepción del médico de la actividad de la enfermedad ${ }^{4}$. OMERACT (Outcome Measures in Rheumatology) y GRAPPA (Group for Research and Assessment of Psoriasis and Psoriatic Arthritis) recomiendan la evaluación de seis dominios en pacientes con APs: recuento articular periférico, piel, función física, calidad de vida, dolor y evaluación global de la enfermedad por el paciente ${ }^{5}$. Otros aspectos importantes de la evaluación son la presencia de entesitis, dactilitis, fatiga, compromiso ungueal, axial, reactantes de fase aguda (RFA) y la evaluación global por el médico ${ }^{6}$. Dandorfer y cols. concluyen que el $50 \%$ de la carga de la enfermedad en APs se debe a los síntomas reumatológicos, mientras que el $50 \%$ restante está constituido por el compromiso cutáneo y los síntomas sistémicos incluyendo a la fatiga ${ }^{7}$.

Varios estudios en Artritis Reumatoidea (AR) encontraron discordancias en las variables consideradas para establecer la evaluación global de la enfermedad por pacientes y médicos ${ }^{8,9,10}$. En APs está información es limitada, Eder y cols. encontraron discordancia entre la evaluación de la enfermedad por médico y paciente, y los factores que contribuyeron de manera más importante a esta diferencia fueron fatiga, dolor, discapacidad y número de articulaciones dolorosas y tumefactas. ${ }^{4}$. Otro estudio reportó que los médicos tienden a colocar puntuaciones más bajas concluyendo que esto pondría de manifiesto la necesidad de incorporar medidas reportadas por el paciente en la evaluación de la actividad de la enfermedad'. Estas diferencias en las estimaciones de la EGEp y EGEm podrían reflejar insatisfacción del paciente, baja adherencia al tratamiento y peores resultados en el control de la enfermedad. Por este motivo, nos propusimos evaluar los factores que influyen en las diferencias entre la evaluación global del médico y del paciente en la APs.

\section{Materiales y métodos}

Realizamos un estudio de corte transversal, incluyendo pacientes $\geq 18$ años, de ambos sexos con diagnóstico de APs según criterios CASPAR ${ }^{11}$ pertenecientes a la cohorte
RAPSODIA (Registro de Artritis Psoriásica del IREP Argentina). Se excluyeron aquellos pacientes que por algún impedimento físico o psíquico no pudieran realizar los cuestionarios (analfabetos, ciegos) y los que cumplían criterios ACR para fibromialgia ${ }^{12}$. Se recolectaron datos sociodemográficos, como sexo, edad, estado civil, escolaridad, ocupación actual y cobertura social. También se consignaron la presencia de comorbilidades y el tratamiento actual. En el examen físico se evaluó el número de articulaciones dolorosas (68) y tumefactas $(66)^{13}$. La Psoriasis cutánea se valoró por PASI (Psoriasis Area Severity Index $)^{14}$. Se registró la eritrosedimentación (ERS $\mathrm{mm} / \mathrm{h}$ ) como reactante de fase aguda (RFA), realizada dentro de la semana previa a la visita. Se evaluaron rigidez matinal, dolor, evaluación global de la enfermedad por el paciente (EGEp) y el médico (EGEm) por medio de Escala Visual Análoga (EVA 0-10 centímetros). Se realizaron autocuestionarios para evaluar calidad de vida: ASQoL (Ankylosing Spondylitis Quality of Life) ${ }^{15}$ y PsAQoL (Psoriatic Arthritis Quality of Life) ${ }^{16}$, para capacidad funcional: HAQ-A (Health Assessment Questionnaireversión Argentina) ${ }^{17}$ y BASFI (Bath Ankylosing Spondylitis Functional Index $)^{18}$ y para actividad de la enfermedad: BASDAI (Bath Ankylosing Spondylitis Disease Activity Index $)^{19}$. Se calcularon los índices compuestos DAS28 (Disease Activity Score) ${ }^{20}$, CPDAI (Composite Psoriatic Disease Activity Index) ${ }^{21}$ y DAPSA (Disease Activity for Psoriatic Arthritis) ${ }^{22}$ el cual sólo pudo realizarse en 56 pacientes ya que en muchos casos no se pudo contar con los resultados de la Proteína C Reactiva (PCR).

Análisis estadístico: Las variables categóricas se expresaron en frecuencia y porcentaje, y las continuas en mediana (m) y rango intercuartilo (RIC). Para comparar variables continuas utilizamos test de T Student y ANOVA y para las variables categóricas test $\mathrm{de} \mathrm{Chi}^{2} \mathrm{y}$ test exacto de Fisher. Correlaciones por test de Pearson. Análisis de regresión lineal múltiple utilizando como variables dependientes EGEm y EGEp. Análisis de regresión logística múltiple utilizando Minimal Disease Activity (MDA) como variable dependiente. Una $\mathrm{p}<0,05$ fue considerada significativa.

\section{Resultados}

Se incluyeron 110 pacientes, 56 varones $(50,9 \%)$, con una edad mediana de 55 años (RIC 45-63) y un tiempo mediano de evolución de la enfermedad de 10 años (RIC 6-17). 56 pacientes $(50,9 \%)$ presentaban compromiso periférico, $2(1,9 \%)$ axial y $52(47,2 \%)$ mixto. En cuanto al tratamiento, 79 pacientes $(71,8 \%)$ recibían AINEs, 89 $(80,9 \%)$ drogas modificadoras de la enfermedad (DME) y $17(15,5 \%)$ agentes anti-TNF $\alpha .24$ pacientes $(21,8 \%)$ cumplían criterios de MDA. Las medidas de evaluación de los pacientes se presentan en la Tabla 1. 


\begin{tabular}{|l|c|}
\hline Variable & $\begin{array}{c}n=110 \\
\boldsymbol{m}(\mathrm{RIC})\end{array}$ \\
\hline EGEp cm & $4,25(2,13-7)$ \\
\hline EGEm cm & $3(1,13-5)$ \\
\hline Dolor por EVA cm & $5(2,7-6)$ \\
\hline HAQ-A & $0,75(0,16-1,22)$ \\
\hline BASDAI & $4,37(1,83-6,53)$ \\
\hline BASFI & $3,55(0,92-5,8)$ \\
\hline PSAQoL & $6(1-12)$ \\
\hline PASI & $1,6(0,4-4,48)$ \\
\hline BSA & $0,75(0-4)$ \\
\hline ERS (mm/hr) & $20,5(8,75-29,25)$ \\
\hline
\end{tabular}

EGEp: Evaluación Global de la Enfermedad por el paciente; EGEm: Evaluación Global de la Enfermedad por el médico; EVA: Escala Visual Análoga; HAQ-A: Health Assessment Questionnaire- versión Argentina; BASDAl: Bath Ankylosing Spondylitis Disease Activity Index; BASFI: Bath Ankylosing Spondylitis Functional Index; PsAQoL: Psoriatic Arthritis Quality of Life; PASI: Psoriasis Area Severity Index; BSA: Body Surface Area; ERS: Eritrosedimentación; RIC: Rango Intercuartilo.

\section{Tabla 1. Medidas de evaluación en 110 pacientes con artritis} psoriásica.

Las mediana de EGEp fue de 4,25 cm (RIC 2,13-7) y de EGEm $3 \mathrm{~cm}$ (RIC 1,13-5). La EGEp tuvo muy buena correlación con dolor, capacidad funcional y actividad de la enfermedad, aceptable con calidad de vida, pero no correlacionó con número de articulaciones tumefactas, dolorosas, PASI, ni ERS (Tabla 2).

\begin{tabular}{|l|c|c|}
\hline \multirow{2}{*}{ Variable } & \multicolumn{2}{|c|}{ EGEp (EVA) } \\
\cline { 2 - 3 } Dolor (EVA) & $\mathbf{r}$ & $\mathbf{P}$ \\
\hline BASDAI & $\mathbf{0 , 7 6}$ & $\mathbf{0 , 0 0 0 1}$ \\
\hline BASFI & $\mathbf{0 , 7}$ & $\mathbf{0 , 0 0 0 1}$ \\
\hline PsAQoL & $\mathbf{0 , 7}$ & $\mathbf{0 , 0 0 0 1}$ \\
\hline Número de articulaciones tumefactas & $\mathbf{0 , 5 6}$ & $\mathbf{0 , 0 0 0 1}$ \\
\hline Número de articulaciones dolorosas & $-0,04$ & 0,744 \\
\hline PASI & 0,05 & 0,705 \\
\hline ERS & $-0,02$ & 0,84 \\
\hline Escolaridad & 0,14 & 0,227 \\
\hline
\end{tabular}

EVA: Escala Visual Análoga; BASDAl: Bath Ankylosing Spondylitis Disease Activity Index; BASFI: Bath Ankylosing Spondylitis Functional Index; PsAQoL: Psoriatic Arthritis Quality of Life; PASI: Psoriasis Area Severity Index; ERS: Eritrosedimentación; EGEp: Evaluación Global de la Enfermedad por el paciente.

Tabla 2. Correlación de EGEp con otras medidas de evaluación y escolaridad.

Por el contrario, la EGEm tuvo buena correlación con medidas de dolor y actividad de la enfermedad, aceptable con capacidad funcional, medidas del examen físico y calidad de vida pero no correlacionó con ERS (Tabla 3).

Se encontraron diferencias significativas tanto en la EGEp y la EGEm entre los pacientes que cumplían y que no cumplían MDA $(1,58 \pm 1,62$ vs $6,38 \pm 8,37 \mathrm{p}=0,015$ y $1,26 \pm 1,19$ vs $4,94 \pm 4,23, p=0,0001$, respectivamente). No

\begin{tabular}{|l|c|c|}
\hline \multirow{2}{*}{ Variable } & \multicolumn{2}{|c|}{ EGEm (EVA) } \\
\cline { 2 - 3 } Dolor (EVA) & $\mathbf{r}$ & $\mathbf{P}$ \\
\hline Evaluación global del paciente (EVA) & $\mathbf{0 , 6 5}$ & $\mathbf{0 , 0 0 0 1}$ \\
\hline BASDAI & $\mathbf{0 , 6 4}$ & $\mathbf{0 , 0 0 0 1}$ \\
\hline BASFI & $\mathbf{0 , 6 2}$ & $\mathbf{0 , 0 0 0 1}$ \\
\hline PsAQoL & $\mathbf{0 , 5 9}$ & $\mathbf{0 , 0 0 0 1}$ \\
\hline Número de articulaciones tumefactas & $\mathbf{0 , 4 3}$ & $\mathbf{0 , 0 0 0 1}$ \\
\hline Número de articulaciones dolorosas & $\mathbf{0 , 4 1}$ & $\mathbf{0 , 0 0 0 1}$ \\
\hline PASI & $\mathbf{0 , 2 1}$ & $\mathbf{0 , 0 0 0 1}$ \\
\hline ERS & 0,09 & $\mathbf{0 , 0 3 4}$ \\
\hline Escolaridad & 0,11 & 0,442 \\
\hline
\end{tabular}

EVA: Escala Visual Análoga; BASDAl: Bath Ankylosing Spondylitis Disease Activity Index; BASFI: Bath Ankylosing Spondylitis Functional Index; PSAQoL: Psoriatic Arthritis Quality of Life; PASI: Psoriasis Area Severity Index; ERS: Eritrosedimentación; EGEm: Evaluación Global de la Enfermedad por el médico.

Tabla 3. Correlación de EGEm con otras medidas de evaluación y escolaridad.

encontramos diferencias en la EGEp y EGEm en cuanto a sexo femenino vs masculino $(5,28 \pm 5,47$ vs $4,99 \pm 8,08$, $\mathrm{p}=0,83$ y $3,64 \pm 2,96$ vs $4,06 \pm 4,46, \mathrm{p}=0,56)$ ni desocupación $(3,94 \pm 2,37$ vs $5,23 \pm 7,1 \mathrm{p}=0,61,1,91 \pm 3,25$ vs $4 \pm 3,78, \mathrm{p}=0,13)$. En los modelos de regresión lineal múltiple, utilizando EGEm y EGEp como variables dependientes, el dolor fue la única variable que se asoció independientemente a ambas evaluaciones (Tablas $4 \mathrm{a}$ y b).

\section{Tablas 4. Regresión lineal múltiple.}

\begin{tabular}{|l|c|c|c|}
\hline Variable & Coeficiente $\boldsymbol{\beta}$ & $\mathbf{t}$ & $\mathbf{P}$ \\
\hline Dolor (EVA) & $\mathbf{0 , 4 8 1}$ & $\mathbf{3 , 2 3 3}$ & $\mathbf{0 , 0 0 2}$ \\
\hline Número de articulaciones dolorosas & $-0,017$ & $-0,112$ & 0,912 \\
\hline Número de articulaciones inflamadas & $-0,110$ & $-0,752$ & 0,456 \\
\hline Rigidez matinal & 0,113 & 0,774 & 0,443 \\
\hline HAQ-A & $-0,121$ & $-0,806$ & 0,424 \\
\hline Fatiga & $-0,143$ & $-1,059$ & 0,295 \\
\hline
\end{tabular}

Variable dependiente: EGEp

EVA: Escala Visual Análoga; HAQ-A: Health Assessment Questionnaire- versión Argentina; EGEp: Evaluación Global de la Enfermedad por el paciente.

\section{4a. Variables asociadas a la EGEp}

\begin{tabular}{|l|c|c|c|}
\hline Variable & Coeficiente $\beta$ & $\mathbf{t}$ & $\mathbf{P}$ \\
\hline Dolor (EVA) & $\mathbf{0 , 5 2 9}$ & $\mathbf{3 , 8 2 6}$ & $\mathbf{0 , 0 0 1}$ \\
\hline Número de articulaciones dolorosas & 0,071 & 0,508 & 0,614 \\
\hline Número de articulaciones inflamadas & 0,048 & 0,351 & 0,727 \\
\hline Rigidez matinal & 0,174 & 1,286 & 0,205 \\
\hline HAQ-A & $-0,126$ & $-0,904$ & 0,371 \\
\hline Fatiga & $-0,193$ & $-1,540$ & 0,130 \\
\hline
\end{tabular}

Variable dependiente: EGEm

EVA: Escala Visual Análoga; HAQ-A: Health Assessment Questionnaire- versión Argentina; EGEm: Evaluación Global de la Enfermedad por el médico.

\section{4b. Variables asociadas a la EGEm}


En la regresión logística, utilizando la presencia de MDA como variable dependiente, tanto la EGEp como la EGEm influyeron significativamente sobre la misma (Tabla 5). Sin embargo la EGEp tuvo una asociación más fuerte que la del médico.

\begin{tabular}{|l|c|c|c|c|}
\hline Variable & OR & \multicolumn{2}{|c|}{ IC 95\% } & p \\
\hline EGEp & $\mathbf{0 , 6 1}$ & 0,42 & 0,89 & $\mathbf{0 , 0 1}$ \\
\hline EGEm & $\mathbf{0 , 4 9}$ & 0,29 & 0,87 & $\mathbf{0 , 0 2}$ \\
\hline
\end{tabular}

Variable dependiente: MDA

EGEp: Evaluación Global de la Enfermedad por el paciente; EGEm: Evaluación Global de la Enfermedad por el médico; MDA: Minimal Disease Activity.

Tabla 5. Regresión logística múltiple.

\section{Discusión}

La APs es una enfermedad compleja con síntomas que derivan del compromiso articular periférico, axial, ungueal, cutáneo y sistémico; por lo tanto la evaluación de la actividad de la enfermedad por parte de los médicos puede no reflejar completamente la carga de la enfermedad en los pacientes. Un estudio multicéntrico demostró que la EGEp a través de EVA es una herramienta útil y fiable en la evaluación de pacientes con $\mathrm{APs}^{23}$, además es un método rápido y simple que reflejaría el status global del paciente. En concordancia con estudios previos, encontramos que los pacientes tienden a asignar puntajes más altos que los médicos a la evaluación de la actividad de su enfermedad $4,7,24$. En nuestro estudio, el recuento de articulaciones tumefactas, dolorosas y el compromiso cutáneo fueron las variables de mayor discordancia entre ambas evaluaciones. Dandorfer y cols. concluyeron que la discordancia fue mayor en pacientes de sexo femenino, mayores de 50 años y aquellos con enfermedad temprana ${ }^{7}$; sin embargo, nosotros no encontramos estas asociaciones. Eder y cols. reportaron que el dolor y la fatiga eran los factores más importantes a la hora de explicar la variabilidad entre ambas evaluaciones ${ }^{4}$, si bien en nuestro estudio la presencia de fatiga no tuvo influencia alguna en la EGE, el dolor, en cambio, fue el factor que tuvo mayor influencia tanto en la evaluación del paciente como la del médico.

En este estudio, la EGEp correlacionó con el dolor y los autocuestionarios de capacidad funcional, actividad de la enfermedad y calidad de vida, no así con el recuento articular, compromiso ungueal o RFA, y si bien la EGEm tuvo un desempeño similar, el recuento articular tuvo alguna influencia en su evaluación. Esta discrepancia podría deberse a que el examen articular es realizado por el médico, por lo tanto, podría tener mayor importancia a la hora de evaluar la actividad de la enfermedad. Lubrano y cols. encontraron correlación entre HAQ y BASDAI y EGEp, no así con recuento articular, PCR ni PASI, concluyendo que esta evaluación refleja un estado global de salud y no identifica dominios específicos de la $\mathrm{APs}^{23}$. En nuestro estudio, el dolor fue la única variable que se asoció de manera independiente a ambas evaluaciones. Además, tanto la EGEp como la EGEm discriminaron bien entre pacientes que cumplían criterios de MDA y aquellos que no. Sin embargo, la EGEp presentó una asociación más fuerte con la MDA, probablemente debido a que la misma es uno de los 7 criterios que conforman este estado de la enfermedad.

Este estudio presenta algunas limitaciones: los pacientes no fueron evaluados por un mismo reumatólogo, por lo tanto la evaluación podría haber estado influenciada por los años de experiencia y la experiencia personal de cada profesional. No evaluamos específicamente otras variables que pueden influir sobre la evaluación del paciente como la presencia de depresión y ansiedad. La fatiga no se evaluó a través de cuestionarios específicos. El único reactante de fase aguda considerado fue la ERS, debido a que la PCR no fue medida en todos los pacientes.

En conclusión, la EGEp fue considerablemente mayor a la EGEm. El dolor fue la única variable asociada independientemente al resultado final de ambas. Ambas evaluaciones discriminan bien entre los pacientes que cumplen o no criterios de MDA.

\section{Bibliografía}

1. Arturi A. Diagnóstico en Reumatología y Enfermedades Autoinmunes Sistémicas. Edición Abbvie. Capítulo 8. Pág. 197-202. 2014.

2. Soriano E, Rosa J, Velozo E, Schpilberg M, Imamura P, Díaz J, et al. Incidence and prevalence of psoriatic arthritis in Buenos Aires, Argentina: a 6-year health management organization-based study. Rheumatology 2011;50(4):729-34.

3. Maldonado Cocco J, Citera G. Reumatología. Segunda Edición, Ediciones Azzurras. Capítulo 33. Pág 462-70. 2012.

4. Eder L, Thavaneswaran A, Chandran V, Cook R, Galdman D. Factors explaining the discrepancy between physician and patient global assessment of joint and skin disease activity in psoriatic arthritis patients. Arthritis Care Res 2015;67(2):264-72.

5. Gladman D, Mease PJ, Strand V, Healy P, Helliwell PS, Fitzgerald $\mathrm{O}$, et al. Consensus on a core set of domains for psoriatic arthritis. J Rheumatol 2007;34(5):1167-70.

6. Leung YY, Ho KW, Zhu TY, Tam LS, Kun EW, Li EK, et al. Construct validity of the modified numeric rating scale of patient global assessment in psoriatic arthritis. J Rheumatol 2012;39(4):844-8.

7. Dandorfer SW, Rech J, Manger B, Schett G, Englbrecht M. Differences in the patient's and the physician's perspective of disease in psoriatic arthritis. Semin 
Arthritis Rheum 2012;42(1):32-41.

8. Nicolau G, Yogui MM, Vallochi TL, Gianini RJ, Laurindo IM, Novaes GS. Sources of discrepancy in patient and physician global assessments of rheumatoid arthritis disease activity. J Rheumatol 2004;31(7):12936.

9. Barton JL, Imboden J, Graf J, Glidden D, Yelin $\mathrm{EH}$, Schillinger D. Patient-physician discordance in assessments of global disease severity in rheumatoid arthritis. Arthritis Care Res 2010;62(6):857-64

10. Berkanovic E, Hurwicz ML, Lachenbruch PA. Concordant and discrepant views of patients' physical functioning. Arthritis Care Res 1995;8(2):94-101.

11. Taylor W, Gladman D, Helliwell P, Marchesoni A, Mease P, Mielants H. Classification criteria for psoriatic arthritis: development of new criteria from a large international study. Arthritis Rheum 2006;54(8):266573.

12. Wolfe F, Smythe HA, Yunus MB, Bennett RM, Bombardier C, Goldenberg DL, et al. The American College of Rheumatology 1990 Criteria for the Classification of Fibromyalgia. Report of the Multicenter Criteria Committee. Arthritis Rheum 1990; 33(2):160-72.

13. Gladman D, Mease P, Healy P, Helliwell P, Fitzgerald $\mathrm{O}$, Cauli A, et al. Outcome measures in PsA. J Rheumatol 2007; 34(5): 1159-66.

14. Marks R, Barton SP, Shuttleworth D, Finlay AY. Assessment of disease progression in psoriasis. Arch Dermatol 1989;125 (2):235-40.

15. Doward LC, Spoorenberg A, Cook SA, Whaley D, Helliwell PS, Kay LJ, et al. Development of the ASQoL: a quality of life instrument specific to ankylosing spondylitis. Ann Rheum Dis 2003;62(1):20-6.

16. McKenna SP, Doward LC, Whalley D, Tennant A, Emery P, Veale DJ. Development of the PsAQoL: a quality of life instrument specific to psoriatic arthritis. Ann Rheum Dis 2004;63(2):162-9.

17. Citera G, Arriola MS, Maldonado Cocco JA, Rosemffet MG, Sánchez MM, Goñi MA, et al. Validation and crosscultural adaptation of an Argentine Spanish version of the health assessment questionnaire disability index. J Clin Rheumatol 2004;10(3):110-5.

18. Calin A, Garrett SL, Whitelock H, Kennedy LG, O’Hea J, Mallorie P, et al. A new approach to functional ability in ankylosing spondylitis. J Rheumatol 1994;21(12):2281-5.

19. Garrett S, Jenkinson T, Kennedy LG, Whitelock H, Gaisford P, Calin A. A new approach to defining disease status in ankylosing spondylitis: the Bath Ankylosing Spondylitis Disease Activity Index. J Rheumatol 1994;21(12):2286-91.

20. Felson DT, Anderson JJ, Boers M, Bombardier C,
Chernoff M, Fried B, et al. The American College of Rheumatology preliminary core set of disease activity measures for rheumatoid arthritis clinical trials. The Committee on Outcome Measures in Rheumatoid Arthritis Clinical Trials. Arthritis Rheum 1993;36(6):729-40.

21. Mumtaz A, Gallagher P, Kirby B, Waxman R, Coates LC, Veale JD, et al. Development of a preliminary composite disease activity index in psoriatic arthritis. Ann Rheum Dis 2011;70(2):272-7.

22. Schoels M, Aletaha D, Funovits J, Kavanaugh A, Baker D, Smolen JS. Application of the DAREA/DAPSA score for assessment of disease activity in psoriatic arthritis. Ann Rheum Dis 2010;69(8):1441-7.

23. Cauli A, Gladman DD, Mathieu A, Olivieri I, Porru G, Tak PP, et al. Patient global assessment in psoriatic arthritis: a multicenter GRAPPA and OMERACT study. J Rheumatol 2011;38 (5):898-903.

24. Lubrano E, Perrotta FM, Parsons WJ, Marchesoni A. Patient's Global Assessment as an Outcome Measure for Psoriatic Arthritis in Clinical Practice: A Surrogate for Measuring Low Disease Activity?. J Rheumatol 2015; 42 (12): 2332-8. 